\title{
CONOCIMIENTO Y ACTITUD DE LA LACTANCIA MATERNA EXCLUSIVA DE MADRES ADOLESCENTES CON NIÑOS MENORES DE SEIS MESES.
}

\author{
KNOWLEDGE AND ATTITUDES ON EXCLUSIVE BREASTFEEDING OF \\ ADOLESCENT MOTHERS WITH CHILDREN UNDER SIX MONTHS.
}

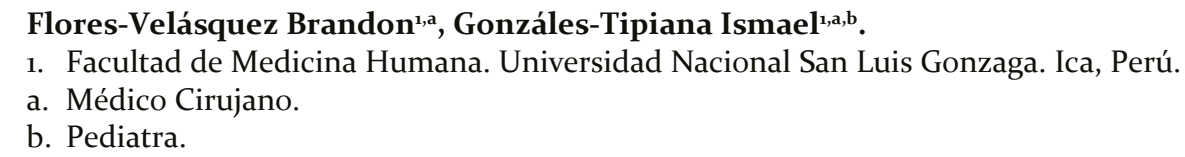

\section{Correspondencia:}

Flores Velásquez Brandon Lui. Dirección: Urb. Las Casuarinas I etapa, Mz.S - 19 Número de celular: 955904238 Correo Electrónico: bradflovelqz@gmail.com

\section{Contribuciones De Autoría:}

FVB participó en la concepción, diseño del artículo, análisis e interpretación de resultados. GTI participaron en el diseño del artículo e interpretación de resultados. Todos participaron en la redacción del artículo y aprobaron la versión final.

Conflicto De Intereses: No declarados.

Financiamiento: Autofinanciado.

\section{Como Citar}

Flores-Velásquez Brandon. Gonzáles-Tipiana Ismael. Conocimiento y actitud de la lactancia materna exclusiva de madres adolescentes con niños menores de seis meses. Rev méd panacea. 2019; 8(3): 101-107. DOl: https://doi.org/10.35563/rmp.v8i3.193

Recibido: 17 - 08 - 2019

Aceptado: 20 - $09-2019$

Publicado: 22 - 09 - 2019

\section{RESUMEN}

Objetivo: Determinar la relación conocimiento y actitud de la lactancia materna exclusiva en madres adolescentes con niños menores de seis meses que acuden al Centro de Salud "Parcona", Ica 2018. Materiales y métodos: El estudio es de tipo descriptivo - correlacional y corte transversal. La muestra lo conformaron 89 madres adolescentes con niños menores de 06 meses. Para la recolección de datos se aplicó como instrumento un cuestionario a través de la encuesta a las madres previo consentimiento informado que acudían para el control de crecimiento y desarrollo de su niño. Estos datos se introdujeron en una hoja de cálculo Excel y luego fueron transferidos al Programa SPSS. Para el análisis inferencial y la prueba de hipótesis se aplicó el estadístico coeficiente de correlación de Pearson y un nivel de significancia de 95\%, en donde $a=0.05$. Resultados: Del total de madres adolescentes el 59.6\% presenta un nivel de conocimiento medio, el $21.3 \%$ tiene un conocimiento bajo y el $19.1 \%$ conocimiento alto; respecto a la actitud el $61.8 \%$ tiene actitud medianamente favorable, el $25.8 \%$ tienen actitud desfavorable y $12.4 \%$ tiene actitud favorable. Respecto a los componentes de las actitudes en la actitud Cognitiva el 52.8\% tiene una actitud desfavorable, el $24.7 \%$ favorable y $22.5 \%$ medianamente favorable; en la actitud emocional el $68.5 \%$ de madres adolescentes tiene una actitud medianamente favorable, el $29.2 \%$ tiene una actitud desfavorable y $2.2 \%$ una actitud favorable; en lo que respecta a la actitud conductual de las madres adolescentes el $43.8 \%$ presenta una actitud medianamente favorable, el $42.7 \%$ muestra una actitud favorable y $13.5 \%$ desfavorable. Conclusiones: El $59.6 \%$ de madres adolescentes tiene un conocimiento medio y $61.8 \%$ tiene una actitud medianamente favorable el cual indica que hay una estrecha relación de conocimiento y actitud de la lactancia materna exclusiva de las madres adolescentes con niños menores de 6 meses.

Palabras clave: Lactancia materna exclusiva, conocimientos y actitudes, madres adolescentes. (Fuente: DeCS BIREME).

\begin{abstract}
Objective: To determine the relationship between knowledge and attitude of exclusive breastfeeding in adolescent mothers with children under six months who attend the Health Center "Parcona", Ica 2018. Materials and methods: The study is descriptive - correlational and cross-sectional. The sample was made up of 89 teenage mothers with children under 06 months. For the collection of data, a questionnaire was applied as an instrument through the survey of the mothers with prior informed consent that they attended to control their child's growth and development. These data were entered in an Excel spreadsheet and then transferred to the SPSS Program. For the inferential analysis and the hypothesis test, the statistical Pearson correlation coefficient and a level of significance of $95 \%$ were applied, where $a=0.05$. Results: Of the total of adolescent mothers, $59.6 \%$ have a medium level of knowledge, $21.3 \%$ have low knowledge and $19.1 \%$ have high knowledge; In relation to the attitude, $61.8 \%$ have a moderately favorable attitude, $25.8 \%$ have an unfavorable attitude and $12.4 \%$ have a favorable attitude. With respect to the components of the attitudes in the Cognitive attitude, $52.8 \%$ have an unfavorable attitude, $24.7 \%$ favorable and $22.5 \%$ moderately favorable; in the emotional attitude $68.5 \%$ of adolescent mothers have a moderately favorable attitude, $29.2 \%$ have an unfavorable attitude and $2.2 \%$ a favorable attitude; Regarding the behavioral attitude of adolescent mothers, $43.8 \%$ presented a moderately favorable attitude, $42.7 \%$ showed a favorable attitude and $13.5 \%$ unfavorable. Conclusions: $59.6 \%$ of teenage mothers have a medium knowledge and $61.8 \%$ have a moderately favorable attitude which indicates that there is a close relationship of knowledge and attitude of exclusive breastfeeding of teenage mothers with children under 6 months.
\end{abstract}

Keywords: Exclusive breastfeeding, knowledge and attitudes, adolescent mothers (source: MeSH NLM) 


\section{INTRODUCCIÓN}

En la actualidad es de conocimiento universal las propiedades y bondades que posee la lactancia materna, debido a que proporciona al recién nacido los nutrientes necesarios y adecuados para un crecimiento y desarrollo óptimo. (1).

La lactancia materna exclusiva implica que el lactante reciba única y exclusivamente alimentación del pecho materno durante los primeros 6 meses de vida, siendo de fácil digestión y asimilación, proporcionando al recién nacido anticuerpos e inmunidad natural, que serán de mucho beneficio para la madre y el bebe. Pero a pesar de los muchos beneficios que brinda la leche materna para el lactante, las madres no prolongan su duración constituyendo un problema álgido que se ha extendido rápidamente en los países de América Latina, ocasionando consecuencias negativas en los niños y niñas, incrementándose la morbimortalidad infantil y padecer enfermedades crónicas no transmisibles en la etapa adulta. $(1,2,3)$

Las madres adolescentes son propensas a abandonar el amamantamiento, debido a que no han desarrollado la madurez, siendo un grupo muy vulnerable. En nuestro país la lactancia materna sobrepasa los datos en relación con el nivel mundial, aunque en estos últimos años su incremento se ha detenido. (4).

La promoción de la salud promueve cambios en la población ayudando a proteger la salud y prevenir las enfermedades a través de comportamientos, prácticas y estilos de vida saludable. El personal de salud debe promover la lactancia materna en todos los espacios y escenarios, iniciando en los escolares y adolescentes en edad reproductiva. En la actualidad tenemos necesidad de promover la importancia y beneficios de la lactancia materna exclusiva sobre todo en las madres adolescentes, brindándole información y educación muy oportuna en el establecimiento de salud, sobre todo del primer nivel de atención. Los profesionales de la salud y el resto de personal de salud deben ser los verdaderos promotores de la lactancia materna. (5).

Los limitados conocimientos de las madres adolescentes sobre lactancia materna, la compleja etapa de su vida, la inexperiencia y la falta de apoyo familiar han propiciado en estas que abandonen precozmente la lactancia materna exclusiva (6).

\section{MATERIALES Y MÉTODOS}

El estudio fue descriptivo-correlacional y de corte transversal. La muestra lo conformaron 89 madres adolescentes con niños menores de 06 meses. Para la recolección de datos se aplicó como instrumento un cuestionario a través de la encuesta a las madres previo consentimiento informado que acudían para el control de crecimiento y desarrollo de su niño. datos se introdujeron en una hoja de cálculo Excel y luego fueron transferidos al Programa SPSS. Para el análisis inferencial y la prueba de hipótesis se aplicó el estadístico coeficiente de correlación de Pearson y un nivel de significancia de $95 \%$, en donde $a=0.05$.

La población está conformada por 116 madres adolescentes con niños menos de seis meses que acuden al consultorio de crecimiento y desarrollo del niño y niña (CRED) del Centro de Salud "Parcona", del distrito de Parcona, provincia y departamento de Ica. Para realizar el cálculo del tamaño de la muestra, se usó la fórmula para poblaciones finitas: $n=z^{2}$. p. q. $N / d^{2}$ $(\mathrm{N}-1)+\mathrm{z}^{2}$. p. q, donde se consideró el nivel de confianza de $95 \%$ y error del 5\%, donde n: Muestra de una proporción de la población, z : factor de confianza (95\%) y su valor es $1.96, \mathrm{~d}$ : error de estimación (5\%) y su valor es $0.05, p$ : proporción esperada (50\%) y su valor es $0.5, q$ : proporción no esperada y su valor es $(p-1)$ es decir 0,05 N: total de la población.

\section{RESULTADO}

La información recolectada se ordenó en la base de datos del programa Excel, para ser revisada si existía algún cuestionario incompleto y luego se procedió a trasladar los datos a la base del programa estadístico SPSS versión 23.

En el análisis descriptivo se presentó la información en tablas y gráficos con frecuencias absolutas y relativas y para el análisis inferencial y la prueba de hipótesis se aplicó el estadístico coeficiente de correlación de Pearson y un nivel de significancia de $95 \%$, en donde $a=$ 0.05 .

\section{CARATERISTICAS SOCIODEMOGRAFICAS DE MADRES ADOLESCENTES CON NIÑOS MENORES DE 6 MESES (VER TABLA 1)}

Respecto a las características sociodemográficas de las madres adolescentes en estudio se encontró lo siguiente:

- En cuanto a la edad materna; la mayoría fue de 17 años en un 51.7\%, seguido por madres de 16 años con un $21.3 \%$.

- $\quad$ Respecto la condición civil la gran mayoría representada por el $61.8 \%$ fueron madres adolescentes solteras.

- $\quad$ En cuanto a la ocupación el $49.4 \%$ fueron amas de casa seguido por el $36 \%$ madres que aun estudiaban. - $\quad$ Respecto al grado de instrucción el 43.8\% apenas alcanzo la secundaria incompleta, seguida por el $40.4 \%$ de madres con secundaria completa.

- $\quad$ Respecto al número de hijos casi la totalidad representada por el 91\% tuvieron un solo hijo.

- $\quad$ Finalmente, respecto al apoyo familiar el $71.9 \%$ manifestó tener apoyo familiar. 
Tabla 1: Carateristicas sociodemograficas de madres adolescentes con niños menores de 6 meses. C.S. Parcona; Ica-2018.

\begin{tabular}{|c|c|c|c|}
\hline \multirow{2}{*}{\multicolumn{2}{|c|}{$\begin{array}{l}\text { CARACTERISTICAS } \\
\text { SOCIODEMOGRAFICAS }\end{array}$}} & \multicolumn{2}{|c|}{$\mathbf{N}=89$} \\
\hline & & $\mathbf{N}$ & $\%$ \\
\hline \multirow[t]{5}{*}{ Edad materna } & 14 años & 7 & 7.9 \\
\hline & 15 años & 17 & 19.1 \\
\hline & 16 años & 19 & 21.3 \\
\hline & 17 años & 46 & 51.7 \\
\hline & Total & 89 & 100 \\
\hline \multirow[t]{5}{*}{ Condición Civil } & Soltera & 55 & 61.8 \\
\hline & Casada & 13 & 14.6 \\
\hline & Conviviente & 19 & 21.3 \\
\hline & Separada & 2 & 2.2 \\
\hline & Total & 89 & 100 \\
\hline \multirow[t]{5}{*}{ Ocupación } & $\begin{array}{c}\text { Ama de } \\
\text { Casa }\end{array}$ & 44 & 49.4 \\
\hline & Estudiante & 32 & 36 \\
\hline & $\begin{array}{c}\text { Trabajo } \\
\text { independien }\end{array}$ & 6 & 6.7 \\
\hline & $\begin{array}{c}\text { Trabajo } \\
\text { dependiente }\end{array}$ & 7 & 7.9 \\
\hline & Total & 89 & 100 \\
\hline \multirow[t]{7}{*}{ rado de instruccic } & $\begin{array}{c}\text { Primaria } \\
\text { incompleta }\end{array}$ & 1 & 1.1 \\
\hline & $\begin{array}{l}\text { Primaria } \\
\text { completa }\end{array}$ & 4 & 4.5 \\
\hline & $\begin{array}{l}\text { Secundaria } \\
\text { incompleta }\end{array}$ & 39 & 43.8 \\
\hline & $\begin{array}{l}\text { Secundaria } \\
\text { completa }\end{array}$ & 36 & 40.4 \\
\hline & $\begin{array}{l}\text { Superior } \\
\text { técnico }\end{array}$ & 8 & 9 \\
\hline & $\begin{array}{c}\text { Superior } \\
\text { universitario }\end{array}$ & 1 & 1.1 \\
\hline & Total & 89 & 100 \\
\hline \multirow[t]{3}{*}{$\mathbf{N}^{\circ}$ hijos } & 1 hijo & 81 & 91 \\
\hline & 2 hijos & 8 & 9 \\
\hline & Total & 89 & 100 \\
\hline \multirow[t]{3}{*}{ Apoyo Familiar } & $\mathrm{Si}$ & 64 & 71.9 \\
\hline & No & 25 & 28.1 \\
\hline & Total & 89 & 100 \\
\hline
\end{tabular}

\section{CONOCIMIENTO DE LA LACTANCIA MATERNA EXCLUSIVA DE MADRES ADOLESCENTES CON NIÑOS MENORES DE 6 MESES (VER TABLA 2)}

Sobre conocimiento de las madres de adolescentes hacia la lactancia materna exclusiva en el estudio se encontró lo siguiente:

- $\quad$ El $59.6 \%$ de madres adolescentes tienen un conocimiento medio, el $21.3 \%$ tienen conocimiento bajo y el $19.1 \%$ tienen conocimiento alto.

Tabla 2: Conocimiento de la lactancia materna exclusiva de madres adolescentes con niños menores de 6 meses en el c.S. Parcona Ica - 2018.

\begin{tabular}{rcccc|}
$\begin{array}{r}\text { CONOCIMIENTO DE LA } \\
\text { LACTANCIA MATERNA }\end{array}$ & Frecuencia & Porcentaje & $\begin{array}{c}\text { Porcentaje } \\
\text { válido }\end{array}$ & $\begin{array}{c}\text { Porcentaje } \\
\text { acumulado }\end{array}$ \\
BAJO & 19 & 21,3 & 21,3 & 21,3 \\
MEDIO & 53 & 59,6 & 59,6 & 80,9 \\
ALTO & 17 & 19,1 & 19,1 & 100 \\
Total & $\mathbf{8 9}$ & $\mathbf{1 0 0}$ & $\mathbf{1 0 0}$ & \\
& & & & \\
\end{tabular}

\section{ACTITUD DE LA LACTANCIA MATERNA EXCLUSIVA DE MADRES ADOLESCENTES CON NIÑOS MENORES DE 6 MESES (VER TABLA 3)}

Respecto a la actitud de la madre adolescente hacia la lactancia materna exclusiva en el estudio se observa en la tabla 3 lo siguiente:

- $\quad$ El $61.8 \%$ de madres adolescentes tienen actitud medianamente favorable, el $25.8 \%$ tienen actitud desfavorable y el $12.4 \%$ actitud favorable.

Tabla 3: Actitud de la lactancia materna exclusiva de madres adolescentes con niños menores de 6 meses en el c.S. Parcona Ica - 2018.

\begin{tabular}{ccccc}
$\begin{array}{c}\text { ACTITUD DE LA LACTANCIA } \\
\text { MATERNA }\end{array}$ & Frecuencia & Porcentaje & $\begin{array}{c}\text { Porcentaje } \\
\text { válido }\end{array}$ & $\begin{array}{c}\text { Porcentaje } \\
\text { acumulado }\end{array}$ \\
$\begin{array}{c}\text { DESFAVORABLE } \\
\text { MEDIANAMENTE FAVORABLE }\end{array}$ & 23 & 25,8 & 25,8 & 25,8 \\
FAVORABLE & 11 & 12,4 & 12,4 & 100 \\
Total & $\mathbf{8 9}$ & $\mathbf{1 0 0}$ & $\mathbf{1 0 0}$ & \\
\hline
\end{tabular}

ACTITUD COGNITIVA HACIA LA LACTANCIA MATERNA DE MADRES ADOLESCENTES CON NIÑOS MENORES DE 6 MESES (VER TABLA 4)

Respecto a la actitud cognitiva el 52.8\% tiene una actitud desfavorable, $22.5 \%$ medianamente favorable y $24.7 \%$ favorable. 
Tabla 4: actitud cognitiva hacia la lactancia materna de madres adolescentes con niños menores de 6 meses en el c.S. Parcona Ica - 2018.

\begin{tabular}{ccccc}
$\begin{array}{c}\text { ACTITUD } \\
\text { COGNITIVA }\end{array}$ & Frecuencia & Porcentaje & $\begin{array}{c}\text { Porcentaje } \\
\text { válido }\end{array}$ & $\begin{array}{c}\text { Porcentaje } \\
\text { acumulado }\end{array}$ \\
\hline $\begin{array}{c}\text { DESFAVORABLE } \\
\begin{array}{c}\text { MEDIANAMENT } \\
\text { E FAVORABLE }\end{array}\end{array}$ & 47 & 52,8 & 52,8 & 52,8 \\
$\begin{array}{c}\text { FAVORABLE } \\
\text { Total }\end{array}$ & 22 & 22,5 & 22,5 & 75,3 \\
\hline
\end{tabular}

\section{ACTITUD EMOCIONAL HACIA LA LACTANCIA MATERNA DE MADRES ADOLESCENTES CON NIÑOS MENORES DE 6 MESES (VER TABLA 5)}

Respecto a la actitud emocional el 29.2\% tiene una actitud desfavorable, $68.5 \%$ una actitud medianamente favorable y $2.2 \%$ una actitud favorable.

Tabla 5: Actitud emocional hacia la lactancia materna de madres adolescentes con niños menores de 6 meses en el c.S. Parcona Ica - 2018.

\begin{tabular}{ccccc}
$\begin{array}{c}\text { ACTITUD } \\
\text { EMOCIONAL }\end{array}$ & Frecuencia & Porcentaje & $\begin{array}{c}\text { Porcentaje } \\
\text { válido }\end{array}$ & $\begin{array}{c}\text { Porcentaje } \\
\text { acumulado }\end{array}$ \\
$\begin{array}{c}\text { DESFAVORABLE } \\
\text { MEDIANAMENT } \\
\text { E FAVORABLE }\end{array}$ & 26 & 29,2 & 29,2 & 29,2 \\
FAVORABLE & 2 & 68,6 & 68,6 & 97,8 \\
Total & 89 & 100 & 100 & 100 \\
\hline
\end{tabular}

\section{ACTITUD CONDUCTUAL HACIA LA LACTANCIA MATERNA DE MADRES ADOLESCENTES CON NIÑOS MENORES DE 6 MESES (VER TABLA 6)}

Respecto a la actitud conductual el $43.8 \%$ de madres adolescentes tiene una actitud medianamente favorable, $42.7 \%$ favorable y $13.5 \%$ desfavorable.

Tabla 6: actitud conductual hacia la lactancia materna de madres adolescentes con niños menores de 6 meses en el c.S. Parcona Ica - 2018.

\begin{tabular}{ccccc}
$\begin{array}{c}\text { ACTITUD } \\
\text { CONDUCTUAL }\end{array}$ & Frecuencia & Porcentaje & $\begin{array}{c}\text { Porcentaje } \\
\text { válido }\end{array}$ & $\begin{array}{c}\text { Porcentaje } \\
\text { acumulado }\end{array}$ \\
$\begin{array}{c}\text { DESFAVORABLE } \\
\text { MEDIANAMENT }\end{array}$ & 12 & 13,5 & 13,5 & 13,5 \\
$\begin{array}{c}\text { E FAVORABLE } \\
\text { FAVORABLE }\end{array}$ & 39 & 43.8 & 43,8 & 57,3 \\
Total & $\mathbf{8 9}$ & 42,7 & 42,7 & 100 \\
\hline
\end{tabular}

\section{DISCUSIÓN}

La lactancia materna exclusiva garantiza los aportes de nutrientes necesarios y suficientes para el crecimiento y desarrollo del bebé, así como el aporte de defensas para el organismo y establece el vínculo afectivo entre la madre, la niña o niño, el padre y el entorno familiar (9).

El abandono de la lactancia materna exclusiva es un problema de salud pública a nivel mundial y esta podría estar asociada a las madres adolescentes, las que constituyen un grupo etario demográfico muy vulnerable para el abandono de esta práctica esencial en el desarrollo del niño $(7,8)$.

De los datos obtenidos en el estudio realizado se encontró de las características sociodemográficas respecto a la edad de las madres adolescentes prevalece las de 17 años en un 51.7\%. En cuanto al estado civil el $61.8 \%$ de madres es soltera, seguido de un $21.3 \%$ que son convivientes; en lo que respecta a la ocupación el $49.4 \%$ es ama de casa y $36 \%$ son estudiante; referente al grado de instrucción el $43.8 \%$ tienen secundaria incompleta y $40.4 \%$ tienen secundaria completa. El $91 \%$ de madres adolescentes tienen un solo hijo y el $71.9 \%$ cuenta con apoyo familiar.

En el estudio de Toala y Cols. (2) de madres adolescentes del año 2018 el 52\% de adolescente culmino secundaria completa: $53 \%$ es ama de casa y $39 \%$ es estudiante. En estudios de Santos y Cols. (10) respecto al grado de instrucción el $51.9 \%$ de madres adolescentes tienen secundaria incompleta, el $54.3 \%$ son estudiantes y $32.1 \%$ son amas de casa. En otros estudios de Miranda y cols. (1) el $43.5 \%$ eran estudiantes y $40.3 \%$ son amas de casa. Toledo Ruiz (11) en su estudio de conocimientos sobre lactancia materna en madres adolescentes respecto a grado de instrucción el $48 \%$ estudiaron secundaria, en lo que respecta a nivel educativo de madres adolescentes el $72 \%$ son estudiantes y $26 \%$ se dedica a la casa. Según el estudio de Alvares y cols. (12) el $61.7 \%$ de madres adolescentes respecto a ocupación son amas de casa y $29.6 \%$ son estudiantes; Castro y cols. (7) el $80 \%$ de madres adolescentes tiene secundaria, en cuanto a su ocupación $53.3 \%$ es estudiantes y $24 \%$ es ama de casa; en su estudio Delgado y cols. (13), respecto al grado de instrucción el $68 \%$ de madres adolescentes estudian secundaria, y según ocupación el $53.2 \%$ son amas de casa y $19.4 \%$ se dedican a su hogar. En el estudio de Flores Pisconte (18) la edad que prevalece de madres adolescentes es de 17 años con un $61.1 \%$, el nivel de escolaridad fue de $88.9 \%$ secundaria y en lo que respecta a ocupación el $50 \%$ son estudiantes y $33.3 \%$ son amas de casa.

En el estudio sobre nivel de conocimientos sobre la lactancia materna exclusiva de madres adolescentes el $59.6 \%$ tiene un conocimiento medio; el $21.3 \%$ tienen un conocimiento bajo y el $19.1 \%$ un conocimiento alto. 
Estos datos coinciden con los estudios de Romero y cols. (10) refiere que el $58 \%$ de madres adolescentes tienen conocimiento medio, el $22.2 \%$ tiene conocimiento bajo y $19.8 \%$ tiene conocimiento alto.

Los datos obtenidos tienen cierta relación con estudios de Cuentas y cols. (14) en su estudio de conocimientos el $73.1 \%$ de madres adolescentes tienen un nivel de conocimiento medio, el $15.4 \%$ conocimiento bajo y $11.5 \%$ conocimiento alto.

También en estudios de Coyutupac y cols. (15) en su estudio concluye que el $65 \%$ de madres adolescente tiene conocimiento medio, de igual forma Alvares Peres (12) concluye que el $58 \%$ de madres adolescentes presentan un conocimiento medio sobre la lactancia materna exclusiva. Asimismo, en su estudio Delgado y cols. (13), presenta que el $48.4 \%$ de madres adolescente presentaron un nivel de conocimiento medio y $41.4 \%$ un nivel de conocimiento bajo. El conocimiento es la suma de hechos y principios que se adquieren y retienen a lo largo de la vida como resultado de las experiencias y aprendizaje del sujeto originando cambios en el proceso del pensamiento, acciones o actividades de la persona (16).

Uno de los factores que favorece el éxito de la lactancia materna viene hacer la información oportuna que se brinda a la madre sobre los beneficios y técnica, la que fortalecerá sus conocimientos para sus hijos (5).

En relación con la actitud de las madres adolescentes hacia la lactancia materna en el estudio se observa que del $61.8 \%$ de la actitud es medianamente favorable; $25.8 \%$ tiene una actitud desfavorable y en $12.4 \%$ la actitud es favorable.

En estudios realizados de Cuentas y cols. (14) en madres adolescente el $61.5 \%$ presenta una actitud medianamente favorable; el $30.8 \%$ una actitud desfavorable y $7.7 \%$ tiene una actitud favorable, siendo una debilidad. Encontramos datos similares en un estudio de Castro y cols. (7) respecto a la actitud de las madres adolescentes hacia la lactancia materna que el $56.7 \%$ tenía una actitud medianamente favorable, el $23.3 \%$ una actitud desfavorable y $20.0 \%$ una actitud favorable. La actitud es la disposición positiva o negativa frente a otras personas y cosas que nos rodea. Son comportamientos de las personas que pueden producirse en diversas circunstancias y definidas por sus valores. Son conocimientos sociales que se construyen en base a las experiencias vividas, creencias $y$ sentimientos (5).

En el presente estudio respecto a las dimensiones de las actitudes en lo que se refiere a la actitud Cognitiva el $52.8 \%$ tiene una actitud desfavorable, el $24.7 \%$ favorable y $22.5 \%$ medianamente favorable. Relacionando con un estudio de Castro y cols. (7) nos muestra en la actitud cognitiva el $53.3 \%$ presenta una actitud medianamente favorable, $26.7 \%$ favorable y $20.0 \%$ desfavorable. En otro estudio realizado por Cuentas y Cols (14) en madres adolescentes el $69.2 \%$ tiene una actitud cognitiva medianamente favorable y $30.8 \%$ la actitud cognitiva es desfavorable. Está actitud está referido al conocimiento y sin conocimientos no hay actitud, en él se incluyen "las creencias" acerca de un objeto puede haber creencias estructuradas basadas sobre datos poco reales 0 erróneos (17).

El segundo componente que presentan las madres adolescentes es la actitud emocional, en el presente estudio el $68.5 \%$ tiene una actitud medianamente favorable, el $29.2 \%$ tiene una actitud desfavorable y $2.2 \%$ una actitud favorable. En un estudio de Castro y cols. (7) obtiene datos similares en la actitud afectiva o emocional, el $63.3 \%$ de madres adolescentes presenta una actitud medianamente favorable, $20.0 \%$ desfavorable y $16.7 \%$ favorable. En otro estudio realizado por Cuentas y Cols. (15) en madres adolescentes el $65.4 \%$ tiene una actitud emocional medianamente favorable, el $23.1 \%$ una actitud desfavorable y $11.5 \%$ tiene una actitud favorable. La actitud emocional o afectiva se conoce como un componente muy sentimental lleno de emociones y sentimientos unidos al objeto. Esta característica estará relacionada con las vivencias afectivas y sentimientos de nuestra vida, estas van a provocar un efecto positivo o negativo en relación con un objeto (17).

Finalmente, en el presente estudio tenemos la actitud conductual de las madres adolescentes donde el $43.8 \%$ presenta una actitud medianamente favorable, el $42.7 \%$ muestra una actitud favorable y $13.5 \%$ actitud desfavorable. En un estudio de Castro y cols. (7) obtiene datos similares en la actitud conductual donde el $48.7 \%$ presenta una actitud medianamente favorable, $30.0 \%$ una actitud favorable y $23.3 \%$ una actitud desfavorable. En el estudio de investigación Cuentas y Cols. (14) en madres adolescentes el $65.4 \%$ tiene una actitud medianamente favorable, el $26.9 \%$ una actitud desfavorable y $7.7 \%$ la actitud es favorable. Esta actitud es el comportamental y es una predisposición para actuar que consiste en las acciones que se realizan hacia un objeto en base a la evaluación afectiva que anteriormente se realizó, La actitud conductual es la predisposición de actuar frente al objeto actitudinal caracterizado o evaluado positiva o negativamente (17). 


\section{REFERENCIAS BIBLIOGRÁFICAS}

1. Miranda YC, Umanzor SL. Conocimiento, actitudes y práctica de la lactancia materna exclusiva en mujeres primigestas de 15 a 24 años que acuden a consulta al centro de salud Francisco Buitrago. Abril a mayo 2014. [Tesis para optar título de Médico Cirujano]. Managua: Universidad Nacional Autónoma de Nicaragua; 2015. [accesado 16 octubre del 2017]. Disponible en: http://repositorio.unan.edu.ni/1209.

2. Toala Alvarado DP, Sánchez Guanopatin GD. Conocimiento de la Lactancia Materna en madres adolescentes que acceden a un Hospital del Sur de Guayaquil. [Tesis para optar el Titulo de Licenciada en Enfermería]. Ecuador: Universidad Católica Santiago de Guayaquil; 2018. [Consultado 2018 Julio 12]. Disponible en: http://repositorio.ucsg.edu.ec/bitstream/3317/10116/ 1/T-UCSG-PRE-MED-EN-450.pdf

3. Gutiérrez JL, Vásquez ChD. Conocimientos, actitudes y prácticas de la lactancia materna exclusiva en puérperas adolescentes del hospital primario Jacinto Hernández. Abril a septiembre 2015. [Tesis para optar título de Médico Cirujano]. Managua: Universidad Nacional Autónoma de Nicaragua; 2015. [Consultado 2017 noviembre 28]. Disponible en: http://repositorio.unan.edu.ni/1435/

4. Coronado López N, Vásquez Manuel $\mathrm{Y}$. Conocimientos sobre lactancia materna y prácticas de amamantamiento en madres puérperas adolescentes. [Tesis para optar título de Licenciatura en Enfermería]. Lima: Universidad Ricardo Palma; 2015.

5. Vásquez Delgado R. Conocimientos, actitudes y prácticas de lactancia materna de las madres que acuden al Centro de Salud San Luis. [Trabajo de investigación]. Lima: Universidad Nacional Mayor de San Marcos. [Consultado 2017 Setiembre 18]. Disponible http://ateneo.unmsm.edu.pe/ateneo/bitstream/1234 56789/2477/1/Vasquez_Delgado_Rocio_del_Pilar_201 2.pdf.

6. Pinilla Gómez E, Domínguez Nariño C, García Rueda A. Madres adolescentes, un reto frente a los factores que influyen en la lactancia materna exclusiva. [Trabajo de investigación]. Zaragoza: Universidad de Zaragoza; 2014. [Consultado 2017 diciembre 10. Disponible en: http://zaguan.unizar.es/record/14082/files/TAZ-TFG2014-317.pdf.

7. Castro Huamán R, Ordoñez Torres LL. Actitud frente a la práctica de lactancia materna exclusiva de madres adolescentes que acuden al C. S. Cristóbal. [Tesis para optar título de Licenciado en Enfermería]. Huancavelica: Universidad Nacional de Huancavelica; 2015.

8. Vásquez NM. Conocimientos y prácticas sobre lactancia materna y factores asociados en mujeres en período de lactancia en el área urbana de Cuenca, 2014. [Tesis para la obtención del título de especialista en pediatría]. Cuenca: Universidad de Cuenca; 2016.
[Consultado 2017 diciembre 20]. Disponible en: http://dspace.ucuenca.edu.ec/bitstream/123456789 /23705/1/tesis60.pdf

9. Ministerio de Salud. Guía Técnica de Implementación de Grupos de Apoyo Comunal para Promover y Proteger la lactancia materna exitosa. Lima. 2017.

10. Santos LP, Gubert FA, Sousa FRR, Rêgo GP, Gomes $M A B$, Oriá MOB. Conocimiento, actitud y práctica de madres adolescentes en relación con el amamantamiento materno. Adolesc Saude. 2016; 13(1):7-18.

11. Toledo Ruiz IK. Conocimiento sobre lactancia materna de madres adolescentes con niños menores de 6 meses del Centro de Salud Castillo Grande, Tingo María. [Tesis para optar título de Licenciado en Enfermería]. Huánuco: Universidad de Huánuco; 2016. [Consultado 2017 diciembre 20]. Disponible en: http://repositorio.udh.edu.pe/123456789/478.

12. Álvarez Pérez S, Facho Tejada SR. Conocimientos y actitudes sobre la lactancia materna en madres adolescentes y jóvenes del Hospital Hipólito Unanue. Julio, agosto y septiembre del 2015. [Tesis para optar título de Licenciada en Obstetricia]. Lima: Universidad Privada Norbert Wiener; 2016. [Consultado 2018 enero 30]. Disponible en: http://renati.sunedu.gob.pe/handle/sunedu/41658.

13. Delgado L, Nombera F. Nivel de conocimientos de madres adolescentes en la práctica de la lactancia materna exclusiva Hospital Regional Docente Las Mercedes de Chiclayo. [Tesis para optar el título de Licenciado en Enfermería]. Chiclayo: Universidad Católica Santo Toribio de Mogrovejo; 2013. [Consultado 2018 enero 15]. Disponible en: http://tesis.usat.edu.pe/bitstream/usat/419/1/TL_De IgadoMarruffoLizeth_NomberaPuellesFiorela.pdf.

14. Cuentas Mamani G, Quispe Coaquira N. Conocimientos y Actitudes hacia la lactancia materna exclusiva de madres adolescentes con niños de 0 - 6 meses que acuden al puesto de salud Canchi Grande 2015. [Tesis para optar título de Licenciado en Enfermería]. Juliaca: Universidad Peruana Unión, 2015. [Consultado 2017 noviembre 11]; Disponible en: http://repositorio.upeu.edu.pe/handle/UPEU/243.

15. Cuyutupac Vega AG, Sinche Espinal RI. Nivel de conocimiento sobre lactancia materna de madres adolescentes del Centro de Salud San Agustín de Cajas. [Tesis para optar título de Licenciado en Enfermería]. Huancayo: Universidad Peruana del Centro; 2016. [Consultado 2017 noviembre 15]. Disponible en: http://repositorio.upecen.edu.pe/handle/UPECEN/6 7.

16. Bunge M. Estrategias de la Investigación Científica. Perú: Ed. Fondo Editorial UIGV; 2014.

17. Soto Peña LE. Actitudes y prácticas de Lactancia 
Materna Exclusiva en madres puérperas que acuden al Hospital Antonio Lorena en Curso. [Tesis para optar título de Licenciada en Enfermería]. Cusco: Universidad Andina del Cusco; 2017.

18. Flores Pisconte $C$, Hernandez, Arones E. Conocimiento sobre la importancia de la lactancia materna y práctica en madres adolescentes con niños menores de 6 meses que acuden al Centro de Salud Fonavi IV etapa. [Tesis para optar título de Licenciado en Enfermería]. Ica: Universidad San Luis Gonzaga de Ica; 2014.
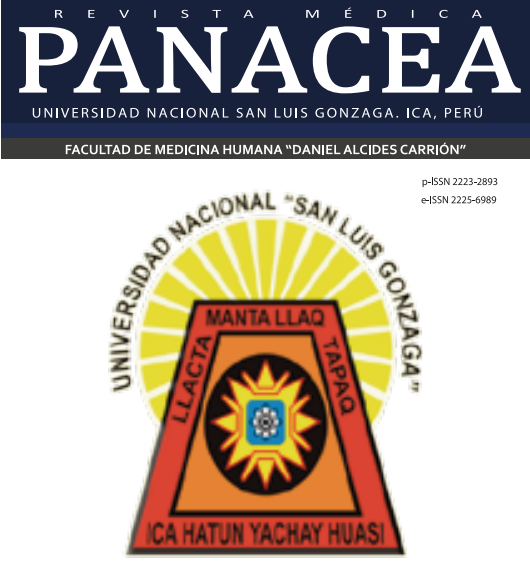

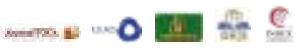

